\title{
Antibiotico - resistenza in Campylobacter jejuni /coli in Italia nel corso del 2002: risultati di una indagine policentrica
}

\author{
Daniele Crotti, Maria Letizia D’Annibale, Mario Spinelli, Fernanda Lanzini, Stefano \\ Rossi, Cinzia Rossi, Rosella Bruno, Maria Luisa Sangiorgio, Marina Bucci \\ Associazione Microbiologi Clinici Italiani, Gruppo di Lavoro “Campyg”, Milano \\ Patterns of in vitro resistances in Campylobacter jejuni/coli in Italy during 2002: a policentric \\ study of AMCLI-Campyg
}

Key Words: Antibiotical resistance, Campylobacter jejuni/coli, Enteritis

\section{SUMMARY}

During 2002, inside Campyg Working Group Project, we examined from 8 italian laboratories the patterns of resistances of 205 strains of Campylobacter spp. These strains were: $88 \%$ C. jejuni (68\% biotype I, 3I\% biotype 2, I\% biotype 3), I I\% C. coli (95.5\% biotype I) and I\% Clo's. We observed these resistances: to erythromycin 3.5\% (2.2\% in C. jejuni, $13.6 \%$ in C. coli), to rokitamicin $2.5 \%$ (I.1\% and $13.6 \%$ respectively), to ciprofloxacin $49.5 \%$ (5I.1\% and 63.6\%), to tetracycline $45.0 \%$ (43.3\% and $59.1 \%$ ), to minocycline $41.6 \%$ (40.0\% and $54.5 \%)$, to gentamycin $0.5 \%$, to chloramphenicole no ones. Some differences concerning frequency of resistances were observed among 8 laboratories, also between $C$. jejuni b. I and C. jejuni b. 2, but these were not significative. Some suggests are given for continuing this surveillance, from an epidemiological point of view too.

\section{INTRODUZIONE}

Nel corso del 1999, un gruppo di Colleghi dell'Associazione Microbiologi Clinici Italiani, coordinati da D. Crotti di Perugia, aveva istituito un Gruppo di Lavoro AMCLI sulle "Infezioni intestinali sostenute da Campylobacter" (GdL Campyg). Precipua finalità di tale Gdl - Campyg era quella di stimolare i colleghi microbiologi a focalizzare la dovuta attenzione sulle infezioni sostenute da Campylobacter spp. e generi strettamente correlati (Clo's), in particolare le specie termotolleranti (o termofile) C. jejuni e C. coli, a qualificare la diagnostica batteriologica al riguardo, e a invitarli, o comunque sensibilizzarli, ad un programma di sorveglianza epidemiologica, al pari di quanto da tempo avviene per Salmonella spp. (ENTERNET, 17).

La distribuzione preliminare di una Monografia al riguardo fornì la base su cui operare in modo omogeneo, standardizzato e ottimale (5).

Tale intento era stato variamente pubblicizzato, e presentato anche ed inizialmente al $10^{\circ}$ Workshop Internazionale su Campylobacter, Helicobacter e microrganismi correlati, tenuto a fine '99 a Baltimora, USA (4).

L'interesse iniziale sembrò reale; numerosi laboratori e centri diagnostici avevano aderito e si erano resi disponibili a collaborare e ad inviare periodicamente i propri risultati al coordinatore dello studio e del GdL - Campyg, o direttamente al Centro di Riferimento Nazionale degli
Enterobatteri, diretto da I. Luzzi presso l'Istituto Superiore di Sanità a Roma. Decollò così un primo Progetto, definito "Campyg Campylobacter - Italia", sulla valutazione delle prevalenze e delle incidenze delle campylobacteriosi nel nostro Paese. A tutti vennero fornite istruzioni scritte più sintetiche, successivamente inserite in una pubblicazione monografica di Microbiologia Medica (3).

Una vivace mole di inziative collaterali prese lo spunto da ciò, e nel corso del 2000 anche un gruppo di Colleghi veterinari operanti essenzialmente in Istituti Zooprofilattici Sperimentali proposero una collaborazione per confrontare quanto accadeva in campo umano e quanto accadeva in campo animale, soprattutto nel settore delle matrici alimentari animali, proponendo, e poi attuando, studi anche più approfonditi in tema di specifiche tipizzazioni molecolari. I risultati, a volte solo parziali, di tali attività, poi proseguite nel corso del biennio 2001-02, furono presentati in varie occasioni congressuali, ed in parte pubblicati (6-9).

Nel corso del 2001, prima di concludere le attività del GdL - Campyg, programmate per la fine del 2002, si è voluto verificare l' andamento delle resistenze in C. jejuni e in C. coli, indubbiamente i principali responsabili di enteriti acute, anche all'interno dei principali biotipi osservabili (quantomeno nel più frequente $C$. jejuni), coinvolgendo direttamente alcuni Laboratori ed alcuni Colleghi 
disposti a cooperare a tal fine, soprattutto alla luce di quanto emerso anche in altri Paesi, ovvero l'incremento delle resistenze, in vitro come in vivo, verso vecchi e nuovi chinoloni, oltrechè verso altre molecole, e verificare al contempo le persistenti basse resistenze nei confronti dei macrolidi $(2,10,13,14,16)$.

\section{MATERIALI E METODI}

Attraverso Notizie AMCLI abbiamo invitato chiunque fosse interessato, a raccogliere i ceppi di Campylobacter isolati da campioni fecali in soggetti con infezione intestinale, e ad inviarli al Laboratorio di Perugia per la identificazione di genere e specie, per la biotipizzazione, per l'esecuzione dell'antibiogramma (anche se già eseguito in loco). Ai Colleghi che hanno aderito sono state rispedite le linee - guida operative che includevano anche le indicazioni per la conservazione ed il trasporto/invio dei ceppi isolati. Di fatto tutti i ceppi sono stati inviati, essenzialmente per posta prioritaria, attraverso un tampone inoculato in provetta agarizzata (Cary Blair, Amies o Stuart, eventualmente al carbone), previa raccolta col medesimo di tutta una patina del ceppo cresciuto in coltura pura, dopo un suo reisolamento su terreno specifico selettivo (ad esclusione del vicino centro di Castiglion del Lago, che ha inviato i suoi ceppi in provetta contenente brodo tioglicolato).

Di fatto, a questo limitato ultimo studio hanno aderito 8 Laboratori italiani, di cui 2 del CentroItalia (Perugia e Castiglion del Lago, PG), 1 del Sud (Larino, CB), e 5 del Nord (Como, Iseo [BS], Domodossola [VB], Chivasso [TO], Paderno Dugnano [MI]).

In tabella 1 riportiamo i ceppi inviati e pervenuti ancora vitali, sui quali è stato possibile poi procedere.
Le identificazioni di genere e di specie e le biotipizzazioni sono state condotte come suggerito previamente $(4,5)$. La valutazione della sensibilità e della resistenza alle molecole antimicrobiche è stata condotta con il sistema di diffusione in agar modificato (Mueller-Hinton al 5\% di sangue di montone). Sono stati saggiati svariati antibiotici. In tale report presentiamo i dati relativi a eritromicina, rokitamicina, acido nalidixico, ciprofloxacina, ofloxacina, norfloxacina, tetraciclina, minociclina, gentamicina, cloramfenicolo.

Tutti i ceppi sono stati poi conservati e inviati al Centro degli Enterobatteri Patogeni dell'Istituto Superiore di Sanità per eventuali ulteriori e più approfonditi tipizzazioni e studi.

Tre ceppi identificati come Clo's (ed uno solo presuntivamente come Arcobacter butzleri) sono stati spediti al Centro di Riferimento EuropeoOMS di Bruxelles (diretto da J. P. Butzler), per verifica e identificazione (18).

Le identificazioni, le speciazioni, le biotipizzazioni, l'antibiogramma, sono stati eseguiti su tutti i 205 ceppi vitali. Dato il numero esiguo dei ceppi provenienti da 4 Centri periferici (tabella 1), ulteriori elaborazioni sono state condotte esclusivamente su 185 ceppi provenienti dai primi 4 Centri riportati in tabella 1. La maggior parte dei ceppi si riferivano a soggetti sintomatici di età pediatrica. Non abbiamo ritenuto utile peraltro in tale lavoro soffermarci su altri aspetti che non riguardassero esclusivamente la identificazione e il comportamento in vitro verso determinate molecole antibiotiche, in particolare quelle di più rilevante eventuale utilizzo clinico (15).

\section{RISULTATI}

Per quanto concerne le identificazioni di genere e di specie, C. jejuni è stato identificato nell' $87.8 \%$

Tabella I. Ceppi inviati dai laboratori partecipanti al centro di Perugia e N. dei ceppi pervenuti

\begin{tabular}{|l|c|c|c|}
\hline \multirow{2}{*}{ LABORATORIO } & N. CEPPI INVIATI & \multicolumn{2}{c|}{ N. DEI CEPPI VITALI } \\
\cline { 2 - 4 } & TOTALI & TOTALI & $\%$ \\
\hline Perugia & 77 & 77 & 100 \\
Castiglion del Lago & 38 & 38 & 100 \\
Como & 40 & 35 & 87.5 \\
Iseo & 38 & 35 & 92.1 \\
Domodossola & $1 \mathrm{7}$ & 7 & 63.6 \\
Chivasso & 8 & 6 & 75 \\
Paderno Dugnano & 6 & 2 & 83.3 \\
Larino & 4 & 205 & 50 \\
\hline TOTALI & 222 & & 92.3 \\
\hline
\end{tabular}


dei casi (180 ceppi), C. coli nel 10.7\% (22 ceppi), Clo's nell'1.5\% (3 ceppi), come riportato in tabella 2 , con variazioni percentuali dal $74.3 \%$ al $100 \%$ per $C$. jejuni, dallo $0 \%$ al $20 \%$ per C. coli. In ambito di biotipizzazione, per quanto riguarda C. jejuni, C. jejuni biotipo 1 è stato prevalente (122 ceppi) rispetto a $C$. jejuni b. 2 (56 ceppi), laddove in 2 soli casi è stato individuato $C$. jejuni b. 3 (rispettivamente $67.8 \%, 31.1 \%$ ed $1.1 \%$ ). Per quanto riguarda $C$. coli, $C$. coli b. 1 ha nettamente prevalso, con 21 ceppi, rispetto all'unico ceppo di $C$. coli b. $2(95.5 \%$ versus $4.5 \%)$. Il tutto è riportato in tabella 3.

In tabella 4 sono riportate le resistenze complessive osservate verso le varie molecole in $C$. jeju$\mathrm{ni} /$ coli. In tabella 5 sono riportate le resistenze dei 4 centri che hanno fornito una casistica adeguata per una loro più attenta valutazione, sia in C. jeju$n i$ (numerosi ceppi), che in $C$. coli (rari ceppi).

Nel complesso, le resistenze ai macrolidi permangono assai contenute: $2.2 \%$ verso eritromicina in C. jejuni e $13.6 \%$ in C. coli $(3.5 \%$ verso Campylobacter spp., ossia C. jejuni/coli), 1.1\% verso rokitamicina in $C$. jejuni e $13.6 \%$ in $C$. coli (2.5\% in Campylobacter spp.). Per contro, anche rispetto ad indagini precedenti, sono aumentate le resistenze verso i chinoloni, sia verso acido nalidixico (solitamente non di impiego terapeutico ma solo diagnostico) che verso i più recenti (va precisato che il comportamento in vitro di ciprofloxacina, ofloxacina e norfloxacina è stato sovrapponibile nel $100 \%$ dei casi), così come verso tetraciclina e minociclina $(10,16,18)$. Nel complesso le resistenze verso ciprofloxacina sono state del $49.5 \%$ (49.4\% in C. jejuni e $50.0 \%$ in $C$. coli), le resistenze verso tetraciclina sono state del $45.0 \%$ ( $43.3 \%$ e $59.1 \%$ rispettivamente), le resistenze verso minociclina del $41.6 \%(40.0 \%$ e $52.5 \%$ ). Infine, mentre verso cloramfenicolo non si sono osservate resistenze, un ceppo di Campylobacter $(0.5 \%)$, ha mostrato una resistenza a gentamicina.

In tabella 6 si riportano invece le resistenze ai principali antibiotici saggiati e di eventuale uso clinico in C. jejuni biotipo 1 C. jejuni biotipo 3 .

\section{DISCUSSIONE E CONCLUSIONI}

Indipendentemente dalle prevalenze di Campylobacter jejuni/coli rispetto a Salmonella spp. o ad altri patogeni enterici, non inerente a tale presentazione, Campylobacter spp, sembra in ogni caso essere presente ed essere responsabile di enterite acuta un po' ovunque $(1,12,18)$. Come da altri Autori riportato, C. jejuni è nettamente prevalente con quasi il $90 \%$ dei casi di campylobacteriosi, con range tra il $74 \%$ circa e il $100 \%$, rispetto a $C$. coli, laddove altre specie sembrano assenti (tabella 2). Indubbiamente interessante è comunque la comparsa di ceppi di "Campylobacter-like organisms", rilevati peraltro in un solo laboratorio. Questo è verosimilmente da imputare al fatto che in tale laboratorio (Perugia) la ricerca di Campylobacter (e simili) viene routinariamente eseguita con la tecnica della membrana filtrante a $37^{\circ} \mathrm{C}$ in terreni non selettivi (11). Ciò può garantire l'isolamento anche di ceppi non appartenenti al gruppo C. jejuni/coli/lari, che nei terreni selettivi specifici dai più usati, eventualmente a $42^{\circ} \mathrm{C}$, tale ceppi "atipici" non possono crescere. In uno di tali 3 casi si ipotizzò Arcobacter butzleri, laddove negli altri 2 casi non fu possibile identificare i ceppi.

In tema di biotipizzazione, come si vede in tabella 3, C. jejuni biotipo 1 prevale su C. jejuni biotipo 3 , con un complessivo $67.8 \%$ contro un $31.1 \%$ (C. jejuni biotipo 3 è rarissimo, $C$. jejuni biotipo 4 non è mai stato osservato). In $C$. coli, è presente pressoché esclusivamente $C$. coli biotipo 1, con il 95.5\% di ceppi (va però detto come pochi siano stati i ceppi di tale specie). Anche qui si osservano delle frequenze variabili. Peraltro, nei principali 4 laboratori partecipanti allo studio, si può osservare come, in fondo, $C$. jejuni biotipo 1 sia di poco prevalente rispetto a $C$. jejuni biotipo 2 . Il perché di tutto questo, e il reale significato di tali biotipi è però tuttora non meglio definito $(4,5)$.

In tema di antibiotico-resistenze, fortunatamente le sensibilità ai macrolidi permangono elevate, con resistenze complessive in vitro del 3.5\% verso eritromicina e $2.5 \%$ verso rokitamicina (molecole rispettivamente a 14 e 16 atomi di C). Come da tempo riportato, C. coli presenta resistenze più elevate rispetto a $C$. jejuni: verso eritromicina $13.6 \%$ versus $2.2 \%$, verso rokitamicina $13.6 \%$ versus $1.1 \%(2,10)$. Per contro le resistente nei confronti dei chinoloni e delle tetra-minocicline sono ulteriormente aumentate $(2,14)$. Non soffermandoci su acido nalidixico (in quanto molecola usualmente utilizzata a scopo diagnostico-identificativo), per quanto riguarda ciprofloxacina (e con questa ofloxacina e norfloxacina, sempre sovrapponibili), si può notare (tabelle 4 e 5) come le resistenze complessive siano praticamente la metà (49.5\%), senza differenze significative, peraltro, tra $C$. jejuni e C. coli. Verso tetraciclina le resistenze totali sono state del $45 \%$, verso minociclina del $41.6 \%$, con leggere maggiori resistenze, anche qui, in C. coli rispetto a C. jejuni $(59.1 \%$ vs $43.3 \%$, e $54.5 \%$ vs $40.0 \%$, rispettivamente). Verso cloramfenicolo non sono emerse ancora resistenze, contrariamente a quanto riportato in altre casistiche internazionali, così come verso gentamicina, nei confronti della quale si è 
Tabella 2. Identificazioni di genere e di specie (complessive e laboratorio per laboratorio)

\begin{tabular}{|l|c|c|c|c|c|c|}
\hline & \multicolumn{2}{|c|}{ Campylobacter jejuni } & \multicolumn{2}{c|}{ Campylobacter coli } & \multicolumn{2}{|c|}{ Clo's } \\
\hline Perugia & 69 & 89.6 & 56.5 & & 3 & 3.9 \\
\hline Castiglion del Lago & 35 & 92.1 & 3 & 7.9 & & \\
\hline Como & 26 & 74.3 & 9 & 25.7 & \\
\hline Iseo & 32 & 91.4 & 3 & 8.6 & \\
\hline Domodossola & 6 & 85.7 & 1 & 14.3 & \\
\hline Chivasso & 6 & 100 & & & \\
\hline Paderno Dugnano & 4 & 80 & 1 & 20 & \\
\hline Larino & 2 & 100 & & & \\
\hline TOTALI & 180 & 87.8 & 22 & 10.7 & \\
\hline
\end{tabular}

Tabella 3. Biotipizzazioni ottenute in C. jejuni e C. coli

\begin{tabular}{|c|c|c|c|c|c|c|c|c|c|c|}
\hline \multirow[b]{2}{*}{ Perugia } & \multicolumn{2}{|c|}{ C. jejuni b. I } & \multicolumn{2}{|c|}{$\begin{array}{ll}\text { C. jejuni } & \text { b. } 2\end{array}$} & \multicolumn{2}{|c|}{ C. jejuni b. 3} & \multicolumn{2}{|c|}{ C. colib. I } & \multicolumn{2}{|c|}{ C. coli b. 2} \\
\hline & 48 & $69.6 \%$ & 21 & $30.4 \%$ & & & 5 & $100 \%$ & & \\
\hline Castiglion d. L. & 19 & $54.3 \%$ & 15 & $42.9 \%$ & I & $2.8 \%$ & 3 & $100 \%$ & & \\
\hline Como & 18 & $69.2 \%$ & 8 & $30.8 \%$ & & & 8 & $88.9 \%$ & I & $11.1 \%$ \\
\hline Iseo & 22 & $68.8 \%$ & 9 & $28.1 \%$ & I & $3.1 \%$ & 3 & $100 \%$ & & \\
\hline Domodossola & 6 & $100 \%$ & & & & & I & $100 \%$ & & \\
\hline Chivasso & 3 & $50 \%$ & 3 & $50 \%$ & & & & & & \\
\hline Paderno D. & 4 & $100 \%$ & & & & & 1 & $100 \%$ & & \\
\hline Larino & 2 & $100 \%$ & & & & & & & & \\
\hline TOTALI & 122 & $67.8 \%$ & 56 & $31.1 \%$ & 2 & $1.1 \%$ & 21 & $95.5 \%$ & I & $0.5 \%$ \\
\hline
\end{tabular}

Tabella 4. Resistenze complessive osservate in $\mathrm{C}$. jejuni/coli

\begin{tabular}{|l|c|c|c|c|c|c|}
\hline \multicolumn{1}{|c|}{ ANTIBIOTICO } & \multicolumn{2}{|c|}{$\begin{array}{c}\text { C. jejuni } \\
\text { I80 ceppi }\end{array}$} & \multicolumn{2}{c|}{$\begin{array}{c}\text { C. coli } \\
22 \text { ceppi }\end{array}$} & \multicolumn{2}{c|}{$\begin{array}{c}\text { C. jejuni/coli } \\
202 \text { ceppi }\end{array}$} \\
\hline Eritromicina & 4 & $2.2 \%$ & 3 & $13.6 \%$ & 7 & $3.5 \%$ \\
Rokitamicina & 2 & $1.1 \%$ & 3 & $13.6 \%$ & 5 & $2.5 \%$ \\
Acido nalidixico & 92 & $51.1 \%$ & 14 & $63.6 \%$ & 106 & $52.5 \%$ \\
Ciprofloxacina & 89 & $49.4 \%$ & 11 & $50.0 \%$ & 100 & $49.5 \%$ \\
Tetraciclina & 78 & $43.3 \%$ & 13 & $59.1 \%$ & 91 & $45.0 \%$ \\
Minociclina & 72 & $40.0 \%$ & 12 & $54.5 \%$ & 84 & $41.6 \%$ \\
Cloramfenicolo & 0 & - & 0 & - & 0 & - \\
Gentamicina & 1 & $0.6 \%$ & 0 & - & 1 & $0.5 \%$ \\
\hline
\end{tabular}

NB: i comportamenti di ofloxacina e norfloxacina sono sempre stati concordanti con quelli di ciprofloxacina. 
Tabella 5 . Percentuale di resistenze osservate nei 4 laboratori con dati più numerosi in C. jejuni e C. coli

\begin{tabular}{|l|c|c|c|c|c|c|c|c}
\hline \multirow{2}{*}{$\%$ DI R } & \multicolumn{2}{|c|}{ PERUGIA } & \multicolumn{2}{c|}{ CASTIGLION D. L. } & \multicolumn{2}{c|}{ COMO } & \multicolumn{2}{c}{ ISEO } \\
\cline { 2 - 10 } & C. jejuni & C.coli & C. jejuni & C. coli & C. jejuni & C. coli & C. jejuni & C. coli \\
\hline Eritromicina & $1.4 \%$ & $0 \%$ & $0 \%$ & $67 \%$ & $3.8 \%$ & $0 \%$ & $3.1 \%$ & $33 \%$ \\
\hline Rokitamicina & $0 \%$ & $0 \%$ & $0 \%$ & $67 \%$ & $3.8 \%$ & $0 \%$ & $3.1 \%$ & $33 \%$ \\
\hline Ciprofloxacina & $46 \%$ & $0 \%$ & $46 \%$ & $100 \%$ & $81 \%$ & $44 \%$ & $31 \%$ & $100 \%$ \\
\hline Tetraciclina & $38 \%$ & $60 \%$ & $46 \%$ & $100 \%$ & $73 \%$ & $56 \%$ & $38 \%$ & $67 \%$ \\
\hline Minociclina & $33 \%$ & $60 \%$ & $46 \%$ & $100 \%$ & $65 \%$ & $44 \%$ & $38 \%$ & $67 \%$ \\
\hline
\end{tabular}

Tabella 6 . Percentuale di resistenze complessive biotipo2 osservate in $\mathrm{C}$. jejuni biotipo I rispetto a $\mathrm{C}$. jejuni

\begin{tabular}{|l|c|c|}
\hline & C. jejuni biotipo I & C. jejuni biotipo 2 \\
\hline Eritromicina & $3.3 \%$ & $0 \%$ \\
\hline Rokitamicina & $1.6 \%$ & $0 \%$ \\
\hline Ciprofloxacina & $47.5 \%$ & $55.4 \%$ \\
\hline Tetraciclina & $45.1 \%$ & $41.1 \%$ \\
\hline Minociclina & $41 \%$ & $39.3 \%$ \\
\hline
\end{tabular}

osservata una sola resistenza in un ceppo di $C$. jejuni.

Nelle tabelle 5 e 6 vengono riportate le percentuali di resistenze osservate nei ceppi inviati da 4 laboratori con maggiori isolamenti di Campylobacter spp. Vi sono alcune interessanti e curiose differenze, nel senso che in alcuni centri le resistenze sono decisamente più elevate, mentre il comportamento tra $C$. jejuni e $C$. coli talora si inverte, ma questo riflette anche, in C. coli, il basso numero di ceppi esaminati.

In tabella 6 vengono invece riportate le resistenze in $C$. jejuni biotipo 1 e in C. jejuni biotipo 2, nei già citati principali 4 centri. Ebbene, non sembrano esservi differenze degne di rilievo, prevalendo le stesse talora in C. jejuni biotipo 1 (nei confronti di macrolidi e tetra-minocicline), tal'altra in $C$. jejuni biotipo 2 (nei confronti dei chinoloni).

Per concludere riteniamo importante perseverare su questa strada, soprattutto a livello istituzionale. Campylobacter spp., ed in particolare i ceppi termoresistenti $C$. jejuni/coli, va sempre ricercato, con i criteri da tempo raccomandati $(4,5)$. L'antibiogramma va sempre eseguito, e va eseguito con il sistema, sia pur leggermente modificato ed adattato, di diffusione in agar. Questo al fine di monitorare la resistenza, sia a fini terapeutici che a fini epidemiologici $(1,2,10,18)$.

Il ruolo che il GdL-Campyg ha avuto è stato quello di sensibilizzare tutti i laboratori diagnostici a questa problematica. Ora è competenza d'altri garantire la continuità in tale direzione.

$\mathrm{Ci}$ auguriamo pertanto che la problematica della gestione delle diarree infettive, sostenute in particolar modo da Campylobacter e generi affini, ma anche da altri vecchi e nuovi patogeni, possa avere sempre maggiore attenzione e riguardo da parte di tutti.

\section{BIBLIOGRAFIA}

1. Allos BM. Campylobacter jejuni infections: update on emerging issues and trends. Food safety. EID 1998; 32: 1201-6.

2. Crotti D. Infezioni intestinali sostenute da Campylobacter jejuni/coli nella seconda metà degli anni '90: aspetti clinico-microbiologici e fenotipi di resistenza. GIMMOC 2002; VI (1): 19-24.

3. Crotti D, Luzzi I. Infezioni da Campylobacter. Microbiol Med 2001; 16: 37-42.

4. Crotti D, Luzzi I, Gomez-Miguel MJ, Piersimoni C. Campylobacter enteritis: an Italian Group for Qualità in Diagnosis and Surveillance. Poster, Abstract in: X International Workshop on CHRO, Baltimore, USA, September 12-19, 1999.

5. Crotti D, Luzzi I, Piersimoni C. Infezioni intestinali da Campylobacter e microrganismi correlati. Caleidoscopio Italiano, Edizioni Medical Systems, 1999.

6. Crotti D, Rossi C, Rossi S, et al. Prospect Campylobacter kit nella diagnosi rapida delle campylobacteriosi: risultati preliminari e indicazioni all'uso. Poster, Abstract in : XXIX Congresso 
Nazionale AMCLI, Rimini, 12-15 settembre 2000.

7. Dionisi AM, Carattoli A, Pezzella C, et al. Campylobacter infection in Italy: human and veterinary surveillance. Poster, Abstract in: XI Internatioal Workshop on CHRO, Freiburg, G, September 1-5, 2001.

8. Dionisi AM, Crotti D, Pezzotti G, et al. Campyg: sorveglianza delle infezioni da Campylobacter in Italia. Microbiol Med 2001; 17: 58-63.

9. Dionisi AM, Ripabelli G, Sammarco ML, Fanelli I, Minelli F, Luzzi I. Caratterizzazione di ceppi di Campylobacter jejuni mediante PCR-RFLP del gene flaA e resistenza agli antimicrobici. Poster, Abstract in: XXX Congresso Nazionale AMCLI, Bari, 12-15 giugno 2001.

10. Engberg J, Aarestrup FM, Taylor DE, Gerner-Smidt $\mathrm{P}$, Nachamkin I. Quinolone and Macrolide Resistance in Campylobacter jejuni and C. coli: Resistance Mechanisms and Trends in Human Isolates. EID 2002; 8: 1501-3.

11. Lopez L, Castillo FJ, Clavel A, Rubio MC. Use a selective medium and a membrane filter method for isolation of Campylobacter species from spanish paediatric patients. Eur J Clin Microbiol Infect Dis 1998; 17: 489-92.

12. Luzzi I, Caprioli A, Tozzi A, et al. ENTERNET 2001: sorveglianza enterobatteri patogeni-Salmonella, VTEC O 157 e Campylobacter. Poster, Abstract in: XXXI Congresso Nazionale AMCLI, Rimini, 17-20 settembre 2002.

13. Nachamkin I, Ung H, Li M. Increasing Fuoroquinolone Resistance in Campylobacter jejuni, Pensylvania, USA, 1982-2001, EID 2002; 8: 1501-3.

14. Piddock LJV. Quinolone resistance and Campylobacter. Clin Microbiol Infect 1999; 5: 23943.

15. Saenz G, Zarazaga M, Lantero M, et al. Antibiotic resistance in Campylobacter strains isolated from animals, foods, and humans in Spain in 1997-1998. Antim Ag Chemoth 2000; 44: 267-71.

16. Sammarco ML, Minelli F, Dionisi AM, Ripabelli G, Luzzi I. Tipizzazione epidemiologica di Campylobacter jejuni mediante "amplified fragment lenght polymorphism" (AFLP). Poster, Abstract in: XXX Congresso Nazionale AMCLI, Bari, 12-15 giugno 2001.

17. Tozzi AE, Gorietti S, Pichiorri T, et al. Sorveglianza delle infezioni da patogeni enterici: l'esempio di ENTERNET. Microbiol Med 2001; 16: 114-7.

18. WHO. The Increasing Incidence of Human Campylobacteriosis. Report and Procedings of a WHO Consultation of Experts. WHO/CDS/CSR/APH 2001.17.

\section{Daniele Crotti}

Sezione di Microbiologia e Parassitologia Clinica,

Ospedale "R. Silvestrini”, via G. Dottori 1, 06156 S. Andrea delle Fratte, Perugia

Tel. 075 5782218; Fax 0755782404

e-mail: analisi.cliniche@ospedale.perugia.it 